\title{
UNA CIUDAD EN BUSCA DE UN GOBIERNO: Una Propuesta para Santiago*
}

\author{
Tomas Chuaqui H., \\ Patricio Valdivieso F. \\ instituto de Ciencia Politica, Universidad Católica de Chile
}

\begin{abstract}
Resumen
Este artículo contiene una propuesta de institucionalidad de gobierno urbano para Santiago que pretende ser politicamente viable. Por ende, se utiliza una estrategia que toma en cuenta la institucionalidad vigente, a la cual se le introducen alteraciones conducentes a la creación de un gobierno propiamente urbano para Santiago. Específicamente, el modelo consiste en potenciar la figura de la Gobernación de la Provincia de Santiago, atribuyéndole funciones de relativa autonomía en cuanto al gobierno de la ciudad. Esta propuesta contempla, además, la creación de un consejo representativo de la ciudadanía que se origina de las alcaldías y consejos municipales. De esta manera, se persigue simultáneamente diseñar una estructura de gobierno urbano que sea políticamente factible, eficiente, y democráticamente representativo. Perseguimos estos propósitos a partir de una revisión del diagnóstico de la circunstancia actual, y proveemos algunos ejemplos comparados de gobierno urbano.
\end{abstract}

\section{Abstract}

This article contains an institutional proposal for urban government in Santiago that considers its political viability. Thus, the strategy followed involves taking into account current institutions, which altered in view of the creation of a properly urban government for Santiago. Specifically, the model consists in a reinforcement of the post of the "Gobernación" of the Province of Santiago, attributing to it functions which entail a greater autonomy in relation to city government. This proposal also considers the creation of a city council with citizenship representation originated at the municipal level. In this way we simultaneously pursue the objective of designing a structure of urban government that ought to be politically viable, efficient, and democratically representative. We pursue these objectives on the basis of a diagnosis of the current state of affairs, and also making use of some comparative examples of urban government.

\section{PALABRAS CLAVE • Gobierno Urbano • Representación • Descentralización • Municipio - Accountability}

\section{INTRODUCCIÓN}

Al igual que otros países de América Latina, Chile enfrenta el gran desafío de mejorar sus instituciones de gobierno y administración interna en beneficio de las condiciones de vida de sus habitantes. Esta necesidad es particularmente apremiante en las grandes áreas urbanas, donde vive la mayor parte de la población chilena ${ }^{1}$, y donde se concentra, por lo tanto, un gran número de demandas ciudadanas que debieran ser procesadas y tener respuestas adecuadas por parte de las estructuras institucionales.

La investigación que dio origen a este estudio fue financiada parcialmente por el Ministerio de Planificación Nacional. Se agradece la activa colaboración del Sr. José Viacava en su calidad de asistente de investigación. También agradecemos los comentarios y sugerencias del Sr. Gustavo Jiménez, y de un árbitro anónimo. Por cierto, el contenido de este escrito es de responsabilidad exclusiva de los autores.

1 Información del último Censo de Población 2002 en http://www.ine.cl/cd2002/index.php 
La agenda del país, por tanto, debe dar prioridad a la mejoría de las estructuras de gobierno y administración de las grandes ciudades. Como se deja ver, no sólo en Chile, sino también en otros países, la política urbana y local tiene un impacto significativo sobre la configuración, consolidación y proyección de agentes y movimientos políticos a nivel nacional. Por ende, el sistema de gobierno urbano cobra una relevancia especial al considerar los equilibrios de poder hacia el interior de los países y los itinerarios a seguir por aquellos que aspiran a acceder a liderazgos políticos.

Este estudio presenta una alternativa de propuesta de gobierno urbano para Santiago, y tal vez extensivo a otras grandes ciudades chilenas, dando énfasis, por un lado, a la factibilidad de reforma dada la realidad política del país, y, por otro, a las consecuencias políticas de tal propuesta. Por cierto, no es el propósito de este trabajo sugerir que todos los problemas que aquejan a las grandes ciudades se resuelven por vía de reforma institucional. Sin embargo, consideramos que la estructura institucional de las grandes ciudades es un factor de gran importancia en tanto condición necesaria para canalizar las demandas ciudadanas por mejores condiciones de vida, y ampliar los espacios de deliberación democrática eficaz.

El método ha consistido en el estudio de la legislación vigente al respecto ${ }^{2}$, realizándose al unísono contactos, a modo de entrevistas y talleres, con actores relevantes según las materias que han ido surgiendo como campo de extensión para la investigación ${ }^{3}$. Del mismo modo, resultó fundamental el acercamiento realizado a destacadas instituciones, tanto estatales como extra-estatales, que tienen líneas de investigación relacionadas con el tema en cuestión.

En estos tiempos somos testigos de transformaciones urbanas que impactan profundamente en las condiciones de vida de las personas. La densificación, la segregación espacial de la población, la contaminación ambiental, la violencia y la inseguridad, la inexorable expansión territorial, entre otros, son todos temas que preocupan a la ciudadanía, y que conforman parte de las externalidades negativas de los asentamientos sociales urbanos. La calidad de vida de los residentes en las metrópolis y zonas similares en Chile -Valparaíso-Viña, Concepción-Talcahuano, Santiago- y otras experiencias cercanas que podrían llamarse ciudades intermedias (por ejemplo, Valdivia y Temuco), no es un problema menor, al menos si se le analiza desde un punto de vista cuantitativo, ya que más del $70 \%$ de la población chilena vive en zonas urbanas. De esta manera, parece ineludible reconocer que los problemas que afectan a las grandes urbes son de hecho problemas nacionales.

El diagnóstico de las metrópolis chilenas es por lo general bastante desfavorable. No obstante las posibilidades que la vida en las grandes ciudades ofrece para el despliegue de múltiples potencialidades humanas, se debe reconocer también que se podrían mejorar las condiciones de las urbes chilenas para acoger una calidad de vida razonablemente digna para todos sus habitantes. Es por esta razón que las grandes urbes son cada día más el centro de atención y preocupación de numerosos investigadores e instituciones académicas y políticas que realizan esfuerzos por entregar un arsenal eficaz de herramientas analíticas y técnicas para combatir los problemas ya indicados.

Constitución Chilena de 1980, leyes orgánicas constitucionales, entre otros.

En el curso de la investigación fueron ejecutados tres talleres de trabajo en el Instituto de Ciencia Política de la Pontificia Universidad Católica de Chile, con personalidades representativas de instituciones gubernamentales, organizaciones gremiales y sectores académicos. Asimismo, se realizó un seminario en el mismo lugar en el que una versión preliminar fue comentada por los Profesores Gert Wagner (Instituto de Economía, PUC); Federico Arenas (Instituto de Geografía, PUC); y Germán Concha (Fundación Jaime Guzmán). 
No obstante los esfuerzos gubernamentales y de actores extra-estatales en las últimas décadas por mejorar las condiciones de vida en las ciudades, problemas tales como la contaminación ambiental, así como la inseguridad ciudadana frente a los delitos, constituyen hoy por hoy problemas cotidianos del ciudadano metropolitano. Aun cuando se cuente con municipios bien estructurados y funcionalmente democráticos, al igual que con intendencias bastante poderosas y articuladas con el resto de los organismos descentralizados o desconcentrados del Estado, el éxito en alcanzar metas satisfactorias en dichas áreas ha sido sólo relativo. Indudablemente esto dice relación con la evolución y el crecimiento natural de las grandes urbes que ocasionan problemas de enormes dimensiones y extremadamente complejos. Pero, precisamente por ello es que tales circunstancias no son impredecibles y, por lo tanto, se hacen exigibles estructuras institucionales de gobierno con mayores capacidades para anticipar eventualidades razonablemente previsibles, y que contribuyan a una mejor interacción entre la sociedad y los agentes e instituciones públicas.

En el contexto indicado, la reforma y modernización del gobierno metropolitano debe apuntar en dos direcciones. Primero, a mejorar la gestión, es decir, la eficiencia y eficacia de la burocracia y de la administración. Segundo, en un contexto democrático, también debe incluir un esfuerzo por incrementar la participación ciudadana y establecer mecanismos de accountability, los cuales tienen efectos sobre la legitimación del gobierno urbano. Estos planos, el de gestión y el de participación, deben coordinarse y no confundirse. No obstante lo benéfica que puede ser la colaboración ciudadana en tanto la resolución de problemáticas propias de la vida en grandes urbes, no es responsabilidad primaria de los ciudadanos el gestionar políticas públicas. La participación ciudadana se remite principalmente, aunque no exclusivamente, al plano político, y no al administrativo o de gestión.

Con el propósito de hacer un aporte constructivo para abordar los desafíos ya indicados, este artículo pone en el debate un modelo-propuesta dirigido a superar algunas de las trabas a la efectiva resolución de problemáticas propias de las zonas metropolitanas: esto es, el diseño de una alternativa de gobierno metropolitano. Dicho sea de paso, el planteamiento no significa necesariamente crear institucionalidad ad-hoc, sino que idear mecanismos a través de los cuales pudiesen coordinarse intersectorialmente las unidades de decisión e implementación -incluyendo las ya existenteshaciendo converger los intereses del Estado, del gobierno subnacional (región o comuna) y los intereses de la comunidad, en torno a la resolución de los problemas. La diferencia entre los estudios y las propuestas que han sido conocidas hasta la fecha y la que presentamos aquí radica, esencialmente, en la entrega de un modelo que nace de los antecedentes existentes y de la consulta a actores pertinentes en la búsqueda de soluciones para las deficiencias detectadas.

Cabe hacer una prevención preliminar: en este estudio no se considera en detalle la posibilidad de crear un Alcalde Mayor de la ciudad elegido popularmente. No obstante sus posibles beneficios en teoría ${ }^{4}$, hemos concluido, en concordancia con la mayor parte de los especialistas consultados, que tal reforma sería inviable dado el contexto jurídico y político de Chile. Particularmente, la circunstancia que condicionó y dificultó sobremanera la figura de un Alcalde Mayor para la ciudad de Santiago es el proceso mismo de transición política desde el autoritarismo al régimen político democrático. Es en este proceso que se inscribe la estructura y gestión democratizada de los municipios, y es precisamente desde los municipios que se perciben las mayores aprensiones y falta de consenso al respecto. Es decir, nuestro sistema de administración interior del Estado fue

Ver por ejemplo, Aylwin (1991:143-146) 
re-articulado en democracia, proceso ocurrido hace tan sólo unos catorce años, y comprendiendo

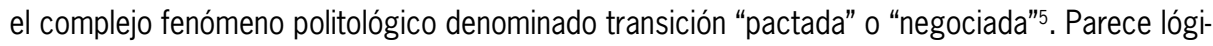
co suponer que dicho proceso tuvo algún corolario en el nivel comunal. En otras palabras, a pesar de que la idea de un Alcalde Mayor para Santiago se propuso a comienzos de los noventa, fue inviable y poco negociable para los actores del sistema político de los primeros años de la transición a la democracia en Chile ceder un poder como el que supone la gestión y liderazgo de un Alcalde Mayor en la ciudad de Santiago ${ }^{6}$. De esta manera, los municipios de la ciudad de Santiago terminaron asumiendo con el transcurso de los años el costo de rechazar la posibilidad de regulación central, realizando ejercicios esporádicos de coordinación metropolitana, cuyo ejemplo más ilustrativo es el asociativismo municipal, pudiendo contabilizarse algunas experiencias muy concretas al respecto, tales como el caso de Empresa Metropolitana de Residuos Sólidos (EMERES) llevado a cabo por algunas comunas de la región metropolitana7.

Por otra parte, desde la perspectiva estrictamente institucional, bajo las condiciones actuales de un Estado unitario y presidencial, y en consecuencia, de los límites que impone la institucionalidad chilena a una profundización de la descentralización -Constitución y Leyes Orgánicas Constitucionales-, este trabajo entra en la discusión sobre la posibilidad de proveer algunas atribuciones nuevas a las municipalidades. Se persigue el propósito de asegurar la inclusión de la representación municipal en el gobierno urbano, lográndose así una adecuada coordinación entre los niveles de administración pública regional, metropolitana y municipal. Si existiese la posibilidad de una reforma constitucional previa sobre la organización del gobierno y administración política de la República, y esa reforma generara condiciones de mayor descentralización local, recién entonces sería pertinente evaluar las alternativas de delegación de funciones de gobierno urbano en los gobiernos comunales. Consideramos, eso sí, que actualmente tal posibilidad es remota.

Teniendo en cuenta las razones expuestas, se ha privilegiado el estudio de posibles adaptaciones de la normativa vigente para satisfacer las necesidades de gobierno urbano, es decir, gestar, a partir de la institucionalidad actual, mecanismos a través de los cuales las funciones de un Alcalde Mayor podrían ser cumplidas, o si se quiere, virtualmente replicadas. A su vez, también se ha buscado, considerando las limitaciones de la organización política actual, potenciar la participación ciudadana, y su concomitante componente de accountability.

La propuesta, expuesta en las conclusiones de este artículo, consiste en deslindar atribuciones del Intendente de la Región Metropolitana y del Gobernador de la Provincia de Santiago, proponiendo que el segundo asuma efectivamente la responsabilidad de la administración de la ciudad. Con el objeto de apoyarlo en la gestión y de constituir un organismo de gobierno democráticamente representativo, proponemos la creación de un Consejo de la Ciudad. Este estaría compuesto por los alcaldes y representantes de concejales de los municipios ubicados en la Provincia de Santiago, y sólo con derecho a voz los miembros del actual Consejo Económico y Social Provincial

La investigación de las transiciones en Schmidt (1995:309-331). Ver también Godoy (1993:79-106).

En otras áreas políticas, donde los gobiernos de la Concertación impulsaron reformas más profundas o de ruptura con la institucionalidad heredada, como habría sido el gobierno de un Alcalde Mayor, el fracaso fue rotundo. Por ejemplo, al comenzar el gobierno del Presidente Aylwin, se propuso el paquete de reformas "Cumplido" que hubiese generado un cambio sustancial en el gobierno del sistema de administración de justicia y que no logró los consensos requeridos en la clase política para ser realizable. Véase Valdivieso y Vargas (2003:169-179).

Subsecretaría de Desarrollo Regional y Administrativo (1999). 
(CESPRO). Consideramos que esta propuesta no implica una alteración radical de la organización político-administrativa del Estado chileno, y por esa razón sería viable políticamente. Pero, al mismo tiempo, creemos que tendría un impacto significativo en la calidad de la gestión y en la representación democrática de los ciudadanos en las grandes urbes, especialmente en Santiago.

\section{ANTECEDENTES DE LA PROPUESTA}

\subsection{Niveles en la administración interior del Estado: mirada funcional y normativa8}

En este apartado daremos cuenta de los distintos niveles de organización y de administración interior del Estado en la actualidad, con el propósito de entender la naturaleza de las problemáticas institucionales que afectan el desarrollo de las grandes ciudades, Santiago en particular. Los ámbitos centrales, regionales-provinciales y comunales serán las instancias de análisis del proceso de "toma de decisiones" y de la interrelación existente entre las diferentes funciones y responsabilidades que le competen a cada uno. Con el propósito de tener claridad al momento de pensar en alternativas de gobierno metropolitano, presentamos a continuación un diagrama de la organización del Estado que muestra, de forma sintetizada, los distintos niveles desde lo nacional a lo local. 


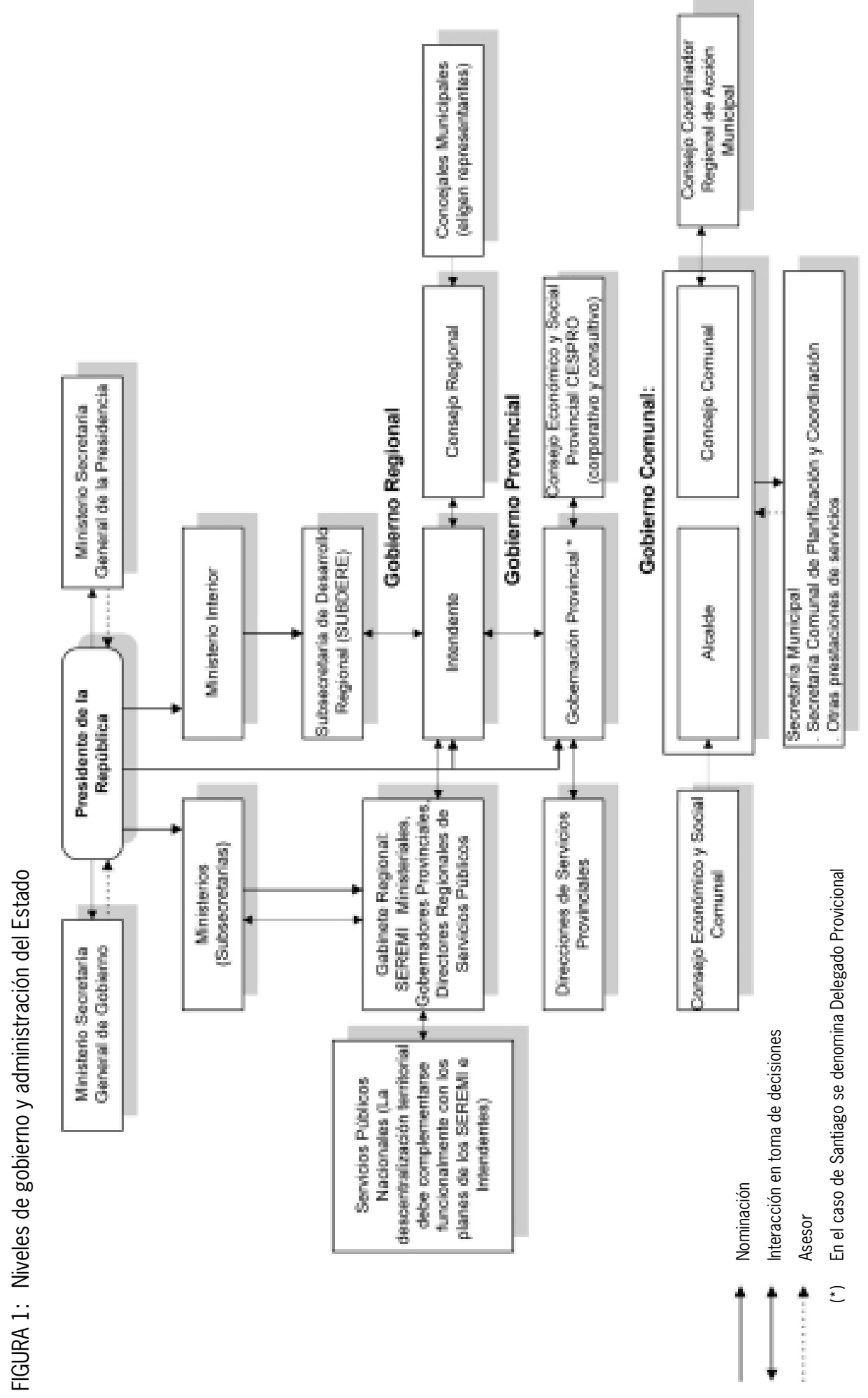


Observando los distintos niveles de la organización de gobierno y administración del Estado chileno, se debe tener en cuenta que el incentivo de los municipios (nivel local más descentralizado) es dar respuesta y satisfacer las necesidades de la comuna, y el mandato de sus representantes proviene de los propios electores locales. Su relación con el gobierno regional, subordinado éste a las estructuras institucionales del gobierno nacional, se basaría en una necesidad de coordinación por parte de este último, y en la entrega de recursos concursables para financiar programas de inversión regional.

Por otra parte, las funciones asignadas a los municipios (sean de áreas metropolitanas o no) tienen la mayor parte de las veces una contraparte no sólo con el gobierno regional, sino que también con otras instituciones del gobierno central. De tal modo que se producen duplicidades y traslapes funcionales y de competencia. Las duplicidades y traslapes tienen como efecto, por una parte, restringir la autonomía de los gobiernos locales y regionales, y, por otra, diluir la responsabilidad en el cumplimiento de sus funciones. Como antecedente, la simple lectura de los artículos №19-25 de la Ley Orgánica Constitucional de Municipalidades №18.695 da lugar a reconocer las siguientes áreas concretas, que provocan confusión:

i) Transporte y Tránsito Público: Respecto a las disposiciones sobre transporte y tránsito público, se producen duplicidades tanto en el Ministerio de Transporte y Telecomunicaciones, como en el Ministerio de Obras Públicas (Dirección de Vialidad), en materias como señalización e instalación de semáforos, entre otros.

ii) Pavimentación y construcción de caminos rurales y urbanos: Se produce duplicidad de funciones entre las Municipalidades y el Servicio de Vivienda y Urbanización (SERVIU), específicamente respecto a la pavimentación de calles. Es facultad del SERVIU la pavimentación y el mantenimiento de calles y vías de libre tránsito ubicadas al interior del límite urbano, pero adicionalmente la ley confiere facultad municipal para realizar esta labor al interior de la comuna con fondos propios.

iii) Protección al Medio Ambiente: Los municipios tienen la tarea de colaborar, junto con otros organismos públicos, en el control de la protección del medio ambiente. Estos organismos son: gobiernos regionales, Servicio de Salud del Ambiente, Comisión de Descontaminación de la Región Metropolitana, etc. Todos los órganos mencionados tienen como función la de proteger el medio ambiente, y aquí radica, por lo tanto, una fuente de duplicidades e interferencias.

iv) Deporte y Recreación: Al igual que los casos anteriores, se dan duplicidades y traslapes en la ejecución de estas facultades, ya que existen distintos organismos autorizados para llevarlas a cabo. En el caso del deporte, los municipios y Dirección General de Deportes y Recreación (DIGEDER) tienen funciones en el campo deportivo.

Claramente, en las materias mencionadas hay fuentes de duplicidades, traslapes e interferencias. En la práctica, estas duplicidades generan conflictos entre los municipios y otras instituciones del sector público, y, al mismo tiempo, originan demoras y dificultades en la satisfacción de las demandas ciudadanas locales.

Adicionalmente, es pertinente referirse a otras características de la forma de articulación de los niveles de administración interior del Estado, así como las unidades subnacionales, en el marco de diversas iniciativas orientadas a promover la descentralización y una mayor gobernabilidad en 
Chile. Debemos destacar que aun cuando se ha buscado la claridad en el actual nivel de descentralización, es un hecho que la complejidad del sistema puede llegar a confundir incluso a los agentes públicos, en cuanto a sus funciones y competencias. $Y$ esto no obstante que se ha establecido, al menos a nivel jurídico, un "régimen que permite a los propios órganos y personas asentados en las regiones intervenir directamente en la solución de los problemas que les son más cercanos, sin esperar respuesta y apoyo del Gobierno Central"9.

Por otra parte, la observación de los niveles de gobierno y administración del Estado evidencian un entramado jurídico subutilizado. Existen instituciones y mecanismos de coordinación que se encuentran atrofiados 0 al menos poco dinamizados, posiblemente como resultado del desconocimiento del sistema en general y de su inherente complejidad. La descoordinación surge, en parte, por la deficiencia de los mecanismos normativos y legales existentes. En la práctica, estos mecanismos, en aquellos casos en que existen, no son susceptibles de utilización eficaz.

Uno de los mayores problemas a sortear por una propuesta de institucionalidad metropolitana en Santiago y otras regiones metropolitanas es la descoordinación entre dos actores institucionales claves del sistema de administración y gobierno interior del Estado: el Gobernador, y los Alcaldes de la Provincia de Santiago. Los focos de ineficacia política y administrativa derivarían de lógicas y racionalidades de funcionamiento no bien articuladas; el Gobernador sigue la lógica de la desconcentración de la autoridad ejecutiva central, desde el Presidente de la República hacia abajo, mientras que el Alcalde sigue la lógica de una entidad descentralizada electa, y ambos deben coexistir, tomar decisiones y administrar, con un marco legal que da origen a duplicaciones, traslapes y "tierras de nadie", al momento de producirse vacíos o problemas concretos.

Al respecto, cabe advertir que la realidad de la ciudad de Santiago no es representativa del resto del país. En el caso de la Región Metropolitana existe una gran complicación, por cuanto no está bien definida, de modo como lo está en el resto de las regiones y provincias, la división de gobierno y de administración que consagra claramente el marco jurídico-legal. El Intendente, en el caso de la Región Metropolitana en particular, ejerce ambas funciones en un solo territorio que, además, es regional. Debemos tener presente que la Provincia de Santiago no tiene gobernación y su importancia sobrepasaría a todos sus socios provinciales. De hecho, creemos que la temática es bastante más compleja y va más allá de la supuesta implementación legal-presupuestaria requerida para materializarla. Hablamos de la incumbencia política-partidista que el tema adquiere si ponemos la mirada de análisis en las posturas e intereses que abrazan los actores involucrados al plantearse esta eventual Gobernación. Esto resulta en una anomalía: "en el caso de la Región Metropolitana, los problemas del país no dejan ver los problemas de la urbe y el efecto capitalidad impacta en el desarrollo nacional, provincial y local. Santiago copa el área Metropolitana y la Región. Se produce el fenómeno de la no-región, de la excesiva cercanía y de la concentración de los excedentes económicos y de las variables democráticas y urbanas"10.

Un paso importante para superar esta situación sería deslindar más precisamente las funciones de gobierno y administración en la ciudad de Santiago. Recordemos que de existir la figura del Gobernador de la Provincia de Santiago, éste debiera velar por el desarrollo provincial y especialmente procurar el funcionamiento de los servicios públicos, así como fiscalizar los programas de infraes- 
tructura económica y social dispuestos en el plan de desarrollo, instrumento crucial en la configuración de políticas acordes a una estrategia tendiente a comprometer a todos los actores del sistema para la consecución de los objetivo comunes. Dadas las características de la Provincia de Santiago, el Gobernador debiera concentrar sus esfuerzos en la administración de los asuntos urbanos.

Siguiendo la línea de tomar conciencia de las condiciones y problemáticas de las estructuras institucionales existentes, se debe considerar que la normativa vigente contempla la facultad de los municipios para celebrar convenios entre sí, materializando las denominadas asociaciones municipales en la procura de una mejor gestión en la prestación de servicios. Y en el plano de la coordinación de éstos, la ley Orgánica Constitucional sobre Gobierno y Administración Regional Nr. 19.175 contempla un Consejo Coordinador Regional de Acción Comunal, integrado por los alcaldes de las comunas comprendidas en la respectiva área. Como se detallará más adelante, éstos y otros elementos abren la posibilidad de razonar en torno a la utilización de aquellos mecanismos e instancias legales ya prescritas y dinamizarlas, imprimiéndoseles la innovación y gestión necesaria, acorde a los lineamientos que la modernización del Estado exige hoy a los componentes del sistema.

\subsection{Gobierno y la administración regional - Región Metropolitana}

Debemos efectuar un análisis de las intenciones destinadas a implementar y ensayar formas novedosas que potencien la administración municipal, los gobiernos regionales o las unidades subnacionales. De hecho, aún no podemos afirmar categóricamente a qué nomenclatura adscribe el desarrollo del proceso descrito hasta este momento. Rescatando alguna polémica que evocamos, algunos ediles han manifestado la disparidad constatada entre los avances de la democracia en el creciente papel protagónico de los municipios y la ancestral competencia administrativa de servicios en crisis que tradicionalmente ha acompañado a estas corporaciones. El clamor de algunas autoridades es transformarlos en verdaderos gobiernos locales con facultades, competencias y recursos necesarios para enfrentar las necesidades de las nuevas comunas.

Aun cuando Chile aparece más descentralizado al nivel comunal, con sus autoridades locales electas, en el nivel regional constatamos la designación del Intendente y la representación indirecta de los Consejeros. Además, los SEREMI asumen pocos compromiso regionales, dado su carácter de unidades desconcentradas de los ministerios nacionales, percibiéndose una doble dependencia y precariedad en su funcionamiento según las disposiciones normativas que muchas veces encubren un traslape de competencias. "En los años noventa, el fortalecimiento del desarrollo municipal y de los gobiernos regionales tiende a completarse con el reclamo persistente de cristalizar en forma creadora los niveles intermedios, acentuar la inter-sectorialidad y la intercomunalidad expandiendo las políticas de participación comunal"11.

Estudiando comparadamente las particularidades del gobierno regional chileno en el contexto latinoamericano, reconocemos que el municipio hoy es considerado un nivel de gobierno, más que de administración de servicios. Del mismo modo, tanto en Chile como en otras latitudes, se ha avanzado hacia la mayor entrega de autonomía, fortaleciendo endógenamente la capacidad institucional y de gestión en la resolución de las necesidades comunales. La elección de sus

11 Ahumada (1998:110). 
autoridades y la convocatoria de la comunidad en los lineamientos estratégicos de desarrollo mejoran y potencian la construcción democrática y social en las comunas ${ }^{12}$. En un plano general, podemos hablar de un proceso sistemático y creciente de descentralización, pues "cada día más se habla de la reinvención del gobierno, reforzando el papel del intendente como articulador regional/provincial de los procesos de desconcentración y descentralización, dándole un fuerte apoyo tecno-político, ya sea a través de la oficina del Intendente; instalando un sistema de toma de decisiones regionales o la presencia asesora de un gerente territorial; redefiniendo el papel del gobernador como activador y coordinador de servicios y municipios; estableciendo gobiernos metropolitanos; creando un sistema de incentivos y premios a la gestión"13. Al mismo tiempo, se reconoce un esfuerzo en mejorar la imagen de regiones y municipios a nivel de sus autoridades para contrarrestar la desafección política que se constata en general dentro del sistema.

Es por todo lo anterior, que los cambios del sistema contenedor que afectan a los niveles de administración y sub-gobiernos nacionales, han transformado y reinventado la gestión de gobierno, cambiando tanto por el lado de la autoridad como por el de los ciudadanos. "La reinvención de éste supone una legitimidad democrática, una nueva gestión en la forma de racionalizar integralmente la oferta y la demanda de bienes y servicios municipales. Se trata de una transición, que va desde el antiguo paradigma de la centralización y unicidad del Estado a la descentralización del mismo"14.

Finalmente, a nivel regional, como es por todos conocido, la institucionalidad ha precedido y generado la regionalidad en términos de identidad. Es decir, la teoría estatista de conformación de lo nacional en América Latina alcanza en el nivel regional también un correlato plausible de aplicar. Sin embargo, "ha llegado el momento de reconocer que los intentos dirigistas y del centro hacia abajo para configurar regiones mediante el artificio de sumar y agregar unidades de la división político-administrativa ha llegado a su fin"15.

Lo anterior fundamenta la aspiración ciudadana y de las autoridades regionales por mayor descentralización política. Es necesario superar la descentralización exclusivamente territorial, pues se requieren decisiones políticas endógenas que no son posibles de alcanzar con la mera desconcentración. Esto incluye, en nuestra opinión, la creación de institucionalidad de gobierno urbano ${ }^{16}$.

Recordando sólo parte de los antecedentes recopilados en este estudio, debemos enfatizar algo que nos parece anómalo. La Región Metropolitana, al ser estudiada comparadamente con el resto de las regiones, comunas y provincias existentes, sobresale de inmediato por una particularidad: la Provincia de Santiago no tiene Gobernación y su importancia sobrepasa a todos sus socios provinciales ${ }^{17}$. El gobierno del Presidente Ricardo Lagos ha reconocido, de alguna manera, la necesidad de darle cabida a la gobernación de Santiago, lo cual es un paso en la dirección correcta, creándose la "Gobernación de Delegación Provincial de Santiago" desde el $1^{\circ}$ de enero de 2001. Hasta ahora, sin embargo, no son completamente claras las responsabilidades y competencias que le corresponden a esta "delegación provincial", y no necesariamente se remiten al

Ahumada (1998:110).

Ahumada (1998:111).

Palma (1995: 54).

Boissier (1994: 181).

Boissier (1994: 187).

Ahumada (1998:111). 
gobierno de la urbe, sino más bien a cuestiones de administración y representación del Intendente en relación a temáticas específicas.

El caso de la Región Metropolitana es complejo, pues confluyen en ella los problemas generales del país. Actualmente, Santiago copa el área metropolitana y la región a la vez, sin sectorizar territorialmente el espacio comprendido ${ }^{18}$. Por su complejidad, tamaño y población, la necesidad de descentralización que tiene la Región Metropolitana es quizás mayor que la de otras regiones. Sin embargo, este simple hecho es de difícil solución.

En efecto, estudiando la composición y las características internas nos encontramos con un espacio que alberga la capital nacional y a la capital metropolitana, así como la concentración del $43 \%$ de la población nacional, del $47 \%$ del PIB, el $60 \%$ del consumo, el $71 \%$ de las grandes empresas, y posee a la vez las comunas más populosas y las más ricas del país.

Si le agregáramos a todo el cuadro descrito con anterioridad, los problemas que cotidianamente se derivan de las externalidades negativas de vivir concentradamente en un territorio y bajo una normativa como la descrita en este estudio, se hace más clara la necesidad de un gobierno urbano para Santiago.

\subsection{Consideraciones adicionales en el debate del tema}

Las aglomeraciones urbanas son el resultado del crecimiento económico y demográfico, que para el caso chileno es un proceso que ha venido desarrollándose con inusitada fuerza en los últimos 20 años. La población comienza a ver en estas oportunidades un gran crisol de actividades y expectativas posibles de extraer algún provecho, sin dimensionar que también acarrea desequilibrios territoriales y sociales propios de la concentración.

La preocupación por el tema metropolitano se ha convertido en una cuestión de alcance estatal, pues las decisiones que se tomen en un territorio con las particularidades ya mencionadas podrían tener repercusiones para los habitantes tanto de la periferia del área metropolitana, así como para el resto de las unidades que conforman el territorio nacional chileno.

Una primera aproximación teórica a los gobiernos metropolitanos señala que en una gran cantidad de casos la descoordinación, desconocimiento en la aplicabilidad de las normas y la falta de voluntad real de las autoridades por solucionar los problemas intersectoriales, llevan a adoptar medidas que idean estructuras metropolitanas configuradas por ley que están por encima de las municipalidades. Lo anterior "obliga a éstas a actuar conjuntamente en una serie de funciones previamente definidas. Este tipo de organización se da en las grandes ciudades de Brasil y Canadá 0 en las grandes comunidades urbanas francesas y suele ser el resultado del crecimiento de la aglomeración más allá de los límites administrativos de la unidad central"19.

En otras ocasiones, el crecimiento nodal alrededor de un núcleo histórico posibilita la formación de municipios metropolitanos, que actúan sin una estructura ni ley por encima de sus competencias. En los dos casos mencionados, la conurbación resultante se transforma por norma en municipio metropolitano. 
En general, podemos señalar que las soluciones más estructuradas, rígidas, y normadas se han desarrollado en países en vías de desarrollo -redemocratizados-, donde un crecimiento urbano rápido y descontrolado ha llevado obligadamente a la formación de organismos especiales que encaucen por ley la realidad urbana. En estas situaciones, no existe el compromiso por cada unidad comunal de intentar solucionar espontánea e intersectorialmente los problemas de la urbe, pues bajo la lógica del desordenado crecimiento urbano por extensión, la conquista del espacio territorial ha sido un logro singular que demanda medidas al nivel central, dejándose de lado la necesaria cooperación, dado que todos los actores concurren en la misma cantidad limitada de recursos o medidas ofrecidas por el nivel superior.

En el caso de la ciudad de Santiago, donde no existe explícitamente una entidad institucional especifica con primeras competencias, se ha optado implícitamente por abrazar una forma de “institucionalización de la aglomeración urbana, que consiste en adaptar ésta a la estructura organizada estatalmente, aprovechando unidades administrativas ya existentes a un nivel superior, como el de la provincia o la región" ${ }^{20}$. Como señalamos anteriormente, la Intendencia y el Gobierno Regional, a través de la delegación provincial, han asumido, en la práctica, las funciones propias de la Gobernación de la Provincia.

La solución a la cual aludimos en el párrafo anterior corresponde sólo a una regionalización del tema metropolitano, al atribuirle a un nivel administrativo superior (Intendencia de la Región Metropolitana, incluidas las cinco gobernaciones provinciales en las que se desconcentra el Intendente) las funciones de coordinación y gestión de la aglomeración urbana, función que consideramos debiera ser propia de la Gobernación de la Provincia de Santiago.

En el sistema actual existe la posibilidad de actuar coordinadamente, funcional e intermunicipalmente para el establecimiento de mecanismos o instituciones dedicados a la prestación de servicios en la metrópolis, como ocurre en Londres, Barcelona, Nueva York y Chicago ${ }^{21}$. En el caso de la Región Metropolitana, la Empresa Metropolitana de Residuos (EMERES) ha sido un ejemplo, ya indicado, de acción concertada de algunos municipios para poner en marcha un sistema de recolección y extracción de basura.

Sin embargo, debemos decir que en general son escasas las áreas metropolitanas que tienen un sistema de competencias bien definido y con recursos suficientes para desarrollarlas. En este sentido, entonces, en algunos casos se ha abusado de la incapacidad de estas unidades para resolver sus problemas, siendo el Estado quien desde "arriba" planifique global y estratégicamente la prestación de servicios y la resolución de los problemas. Se produce, así, un impacto positivo en algún momento inicial de la medida, pero luego los niveles de gobierno local pasan a ser meras entidades administradoras inferiores de las políticas implementadas centralizadamente, quedando inhibida la participación de la comunidad y la proximidad de la gestión del municipio al ciudadano.

Las competencias articuladas supramunicipalmente (gobierno metropolitano, Alcalde Mayor, etc.) o intermunicipalmente (asociatividad municipal) para conformar una organización metropolitana, no son exclusivas, sino que compartidas con otros órganos estatales y locales, por lo que suelen producirse conflictos de intereses entre estas unidades dado que actúan sobre un mismo territo- 
rio. Es así como, por ejemplo, "en París ha habido disputas entre la región y el Estado para el control de la política de transporte"22. En el caso de Santiago las presiones y movimientos sociales que producen conflictos son regularmente resueltos a nivel ministerial del gobierno central, y no por instancias de gobierno, municipal, o provincial. Los intereses en estos casos actualmente sobrepasan con creces cualquier responsabilidad o competencia que puedan tener las autoridades respectivas en un área metropolitana.

Ciertamente deben tomarse en cuenta problemas estrictamente políticos al considerar el funcionamiento de una institucionalidad metropolitana. Así por ejemplo, los municipios expresan una reticencia natural ante la idea de una institucionalidad metropolitana, temiendo perder parte de su autonomía en favor de las instancias superiores o de coordinación. Igualmente, los niveles de la administración superior desarrollan un recelo hacia estas estructuras, pues las consideran inconvenientes y eventualmente una oposición en el caso de que éstas sean representadas por autoridades distintas a la línea política del oficialismo.

Además, la proliferación de nuevos organismos puede ser percibida por algunos como una reburocratización e incremento del gasto público, tendiendo a favorecer, entonces, una hipótesis de reforma mínima, es decir, aprovechar las instituciones y normas jurídicas ya existentes, delegando o transfiriendo mayores competencias y eventualmente recursos a las autoridades en ejercicio, en lugar de gestar una nueva institucionalidad.

Uno de los problemas más graves y recurrentes en la actualidad para el desarrollo de gobiernos metropolitanos efectivos es la desafección ciudadana, pues al generarse un déficit democrático no existe demanda social hacia las autoridades sobre el tema. En el caso chileno, en general, la elección indirecta de Consejeros, así como la designación del Intendente con la dualidad de ser el representante de la región y el ejecutivo del Gobierno Regional, y la desconcentración de la autoridad presidencial, hace que su figura sea sentida como lejana y más allá del control ciudadano.

Además, al no existir cargos gubernativos propios de las ciudades, los partidos políticos, por lo general, no desarrollan agendas públicas para proponer políticas urbanas. Generar institucionalidad urbana representativa podría tener como efecto impulsar a los partidos políticos a generar propuestas para mejorar la calidad de vida de los habitantes de las ciudades. En un país unitario como Chile no es esperable que emerjan partidos regionales, pero sí que se le dé mayor relevancia a los problemas urbanos dentro de los programas políticos de los partidos. Una institucionalidad urbana podría incentivar la canalización de demandas sociales a través de los partidos políticos, cosa que hoy sólo se da parcialmente.

Adicionalmente, desde un punto de vista técnico, será necesario considerar aspectos financieros, uso más eficiente de recursos (costos), y de ordenamiento territorial relativos a cualquier reforma que se quiera implantar. Por ejemplo, aún falta por dirimir la discusión en torno a definir el área metropolitana de Santiago, ya sea como un amplio ámbito regional (adecuado para la planificación estratégica e implementación de instancias coordinadoras superiores) o como un espacio restringido de continuo urbano (adecuado para la cooperación intermunicipal). Como se verá más adelante, en el contexto de nuestra propuesta, trabajamos con el supuesto que la Provincia de Santiago debiera corresponder territorialmente a la metrópolis.

22 Borja (1996: 287). 
Pese a todas las trabas existentes, en suma lo que se persigue es una efectiva gobernabilidad metropolitana. Algunos puntos imprescindibles a considerar para entender la gobernabilidad eventual del sistema metropolitano son los siguientes:

i) Debe existir un nivel estratégico, donde los actores puedan desplegar sus capacidades y visiones.

ii) El nivel metropolitano debe ser un lugar de cooperación para gestionar la promoción de la ciudad, cristalizándose en este nivel la mayoría de las veces como un supermunicipio descentralizado.

iii) El nivel ciudad-municipio es el ámbito político por excelencia, donde se discutirán las propuestas ciudadanas y las consideraciones técnicas pertinentes ${ }^{23}$.

\section{PROPUESTA DE GOBIERNO URBANO PARA LA CIUDAD DE SANTIAGO}

El gobierno y la administración de las áreas metropolitanas sigue siendo un importante desafío para las políticas públicas, que no se resuelve por una planificación sólo de carácter indicativo. Dada la magnitud de los problemas presentados en este estudio, concluimos que es necesaria una reforma institucional. Existen hipótesis de cambio máximo que dicen relación con una modificación institucional a nivel normativo y la correspondiente creación de nuevas instancias de gobierno a nivel metropolitano; hipótesis medias que re-potencian las facultades en las autoridades y estructuras ya provistas por la administración y los niveles subnacionales de gobierno del Estado en Chile, aclarando, a su vez, las competencias correspondientes; $y$, finalmente, hipótesis mínimas que no son otra cosa que la interpretación de las leyes que rigen el proceso, asumiendo las autoridades en forma cabal y plena las responsabilidades señaladas en las leyes y reglamentos.

En primera instancia, nos parece necesaria la creación de una Comisión de Modernización del Gobierno Urbano. Ésta tendría la función de precisar las atribuciones y competencias de los múltiples organismos que se ven involucrados en el gobierno urbano. Como ya se ha visto, existen un sinnúmero de experiencias en las que se distinguen tanto traslapes de competencias, como áreas no cubiertas por ninguna entidad. Esta Comisión tendría que ser coordinada desde el gobierno central. Esta necesidad nos parece independiente de las reformas que se puedan gestar en tanto gobierno urbano, aunque sin duda esta instancia podría cumplir una función importante en la implementación de los cambios correspondientes. Esta entidad debiera apoyarse en un proceso amplio de discusión temática y con la participación de actores provenientes de los distintos niveles de la administración interior del estado, incluyéndose por supuesto al Intendente, los SEREMI, SERPLAC y el Gabinete Regional. Esta discusión debe convocar a los más diversos representantes de organizaciones sociales, ONGs, empresarios, universidades, carabineros, iglesias, estudiantes, etc. También sería positivo considerar la participación de representantes de la comunidad a nivel territorial como por ejemplo parlamentarios, consejeros regionales, consejeros provinciales, Alcaldes, Gobernadores, Juntas de vecinos, etc.

Para empezar, y sin desconocer las particularidades de la circunstancia chilena, nos parece importante considerar modelos de gobierno urbano existentes y relativamente exitosos en otras ciudades 
del mundo. En otros países existen buenos ejemplos de gobiernos políticos y administrativos especiales para la administración de las grandes ciudades. Tal es el caso de Alemania, lugar donde hay básicamente dos modelos a considerar que podrían servir de orientación para pensar en la forma concreta que debiera adoptar el gobierno metropolitano en el caso de Chile, atendiendo a las singularidades legales chilenas ya indicadas ${ }^{24}$. Tal como ocurre en el caso de Chile, en Alemania existen regiones que coinciden con la sede de grandes ciudades (Hamburgo, Berlín y Bremen, por ejemplo). En consecuencia, la estructura político-administrativa para la región y para la ciudad pasa a ser idéntica en la práctica, no obstante que incluyan algunos sectores predominantemente rurales. Sin entrar en detalles respecto a la estructura político-administrativa particular de cada caso, a continuación presentamos dos modelos recurrentes que nos parecen especialmente útiles para argumentar en torno a la factibilidad de un gobierno urbano en nuestro país, y en Santiago, concretamente.

El primer modelo de gobierno consiste en un consejo colegiado, compuesto principalmente por representantes elegidos por la ciudadanía de la región (en el caso de Santiago el equivalente podrían ser los alcaldes y concejales), y un jefe de gobierno, también elegido por la ciudadanía, que preside el consejo y es suprema autoridad político-administrativa de la región/ciudad (en Chile el símil tendría que ser el Intendente Regional o el Gobernador Provincial, aunque éstos no son elegidos popularmente). Los miembros del consejo ocupan comisiones que trabajan en torno a distintos problemas de la región/ciudad, y el consejo también controla y fiscaliza al jefe de gobierno. La principal tarea del jefe de gobierno es coordinar la actividad administrativa de la región/ ciudad, y conciliar el desarrollo metropolitano con el desarrollo nacional. De esta manera, se consigue un equilibrio entre metas nacionales (de las que se preocupa eminentemente el jefe de gobierno, sin perjuicio de su función como actor regional), y el desarrollo adecuado de la región y la urbe (de las que se preocupa prioritariamente el consejo).

El segundo modelo de gobierno consiste en un consejo de representantes de la ciudadanía, y dos autoridades unipersonales ejecutivas, un presidente del consejo y un director. Los miembros del consejo proceden de la elección popular, mientras que las dos autoridades ejecutivas son elegidas por el consejo. El presidente del consejo cumple funciones políticas, dentro y fuera de la región, mientras que el director se concentra en las actividades administrativas de la misma, es decir, se esfuerza por garantizar la buena coordinación administrativa. Como en el modelo anterior, el consejo designa y ocupa comisiones para ver temas específicos de interés regional/urbano, y nombra también autoridades administrativas, que deberán trabajar bajo la supervisión del director.

En el caso de ambos modelos, la estructura administrativa se organiza del siguiente modo: a la cabeza está el jefe administrativo, y después vienen oficinas administrativas con igualdad de rango para tratar temas específicos de la administración regional/urbana: administración general (personal, estadísticas, relaciones públicas, prensa, cuentas); administración financiera (finanzas, caja, impuestos, propiedades, gastos de defensa); derecho, seguridad y orden (judicial, policía y orden público, registro civil, seguros, bomberos, defensa civil); educación y cultura (colegios, eventos culturales); salud y servicios sociales (actividades sociales, jóvenes, deporte, programas de salud y hospitales, compensaciones); obras (obras administrativas, planificación urbana, mediciones, regulaciones urbanas, conjuntos habitacionales, edificaciones, subterráneos, jardines y cementerios); administración de organizaciones públicas (aseo y ornato, mataderos, ferias, siste- 
mas de préstamo, piscinas y baños públicos); administración económica y tráfico (estímulo económico del transporte, estímulo de actividad privada, protección de recursos).

La estructura indicada garantiza una buena coordinación y eficiencia administrativa, y por lo tanto contribuye a una buena gestión, sin que se produzcan los problemas de otros países que no tienen gobiernos regionales/urbanos con autoridades que claramente asuman tareas de coordinación.

La participación ciudadana es otro elemento fundamental para la eficacia del sistema político administrativo. Con independencia de consideraciones teóricas sobre la participación, en la práctica tiene la ventaja de legitimar cualquier medida administrativa y de introducir mayor eficiencia al output. En efecto, la participación garantiza una sintonía entre los servicios del aparato administrativo y las demandas de los usuarios. El uso de los conocimientos y de la actividad de los ciudadanos en beneficio de la actividad administrativa genera condiciones positivas para la eficiencia y eficacia de esa actividad.

En los modelos alemanes ya citados, la participación se practica por vía de la elección directa y de la democracia representativa. Los miembros del Consejo son resultado de elección directa, mientras que en el caso de las autoridades unipersonales ha tendido a predominar un sistema representativo indirecto (elegidos por personas que son representantes de la ciudadanía). Otros elementos de democracia directa son las peticiones, propuestas ciudadanas, y plebiscitos.

La tendencia predominante de la década de 1990 fue de avance de la democracia directa en general. Así, por ejemplo, varios estado-regiones alemanes cambiaron el sistema de elección indirecta por el de elección directa de las cabezas político-administrativas. A su vez, el incremento en participación directa se ha traducido en mayor respaldo y legitimidad de su gestión de coordinación de las actividades administrativas, y por lo tanto mayor eficacia del aparato político-administrativo.

Otra tendencia interesante consiste en incluir la participación ciudadana en varias dimensiones, no sólo en los procesos electorales. Se han afinado mecanismos, tales como plebiscitos y encuestas, con el objeto de priorizar y focalizar mejor la distribución de los recursos comunes, y las organizaciones civiles han asumido papeles protagónicos en la producción misma de los bienes y servicios públicos, tales como salud, educación, seguridad, entre otros. En este sentido, la introducción de mecanismos plebiscitarios y el incentivo de la participación ciudadana en la provisión de bienes y servicios públicos están ligadas: por ejemplo, como resultado de un plebiscito se refuerza la jerarquización de prioridades en el gasto, a la vez que se la legitima. Las iniciativas ciudadanas quedan involucradas en el proceso productivo de bienes y servicios públicos al ser subsidiadas de acuerdo a la jerarquización resultante.

En el marco de este artículo propositivo, ofrecemos agregar a la discusión en curso una propuesta de reforma institucional del gobierno de la ciudad de Santiago, la que posiblemente podría adecuarse también a otras grandes ciudades del país. 
FIGURA 2: Modelo de gobierno de la ciudad de Santiago

\section{Nivel de gobierno y administración regional}

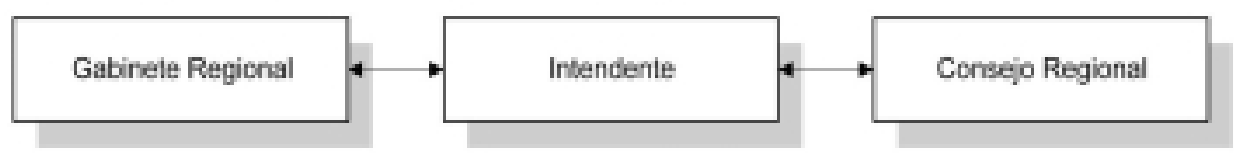

Nivel de gobierno y administración de la ciudad de Santiago (propuesta)

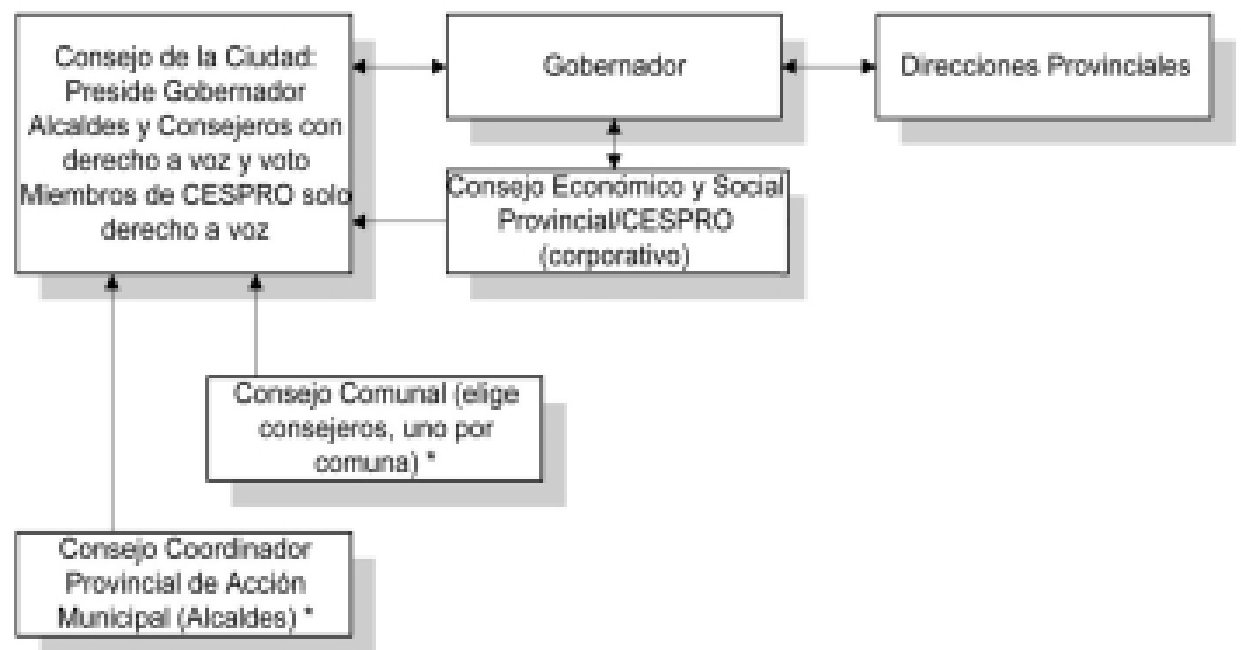

$\left({ }^{*}\right.$ Los miembros del Consejo Comunal y del Consejo Coordinador Provincial de Acción Municipal son de representación popular directa; los Consejos Comunales pertenecen a municipalidades de la Provincia de Santiago; los alcaldes y concejales que participan en el Consejo de la Ciudad provienen de municipios que pertenecen a la Provincia de Santiago. 
FIGURA 3: Niveles de gobierno y administración del Estado, incluida propuesta

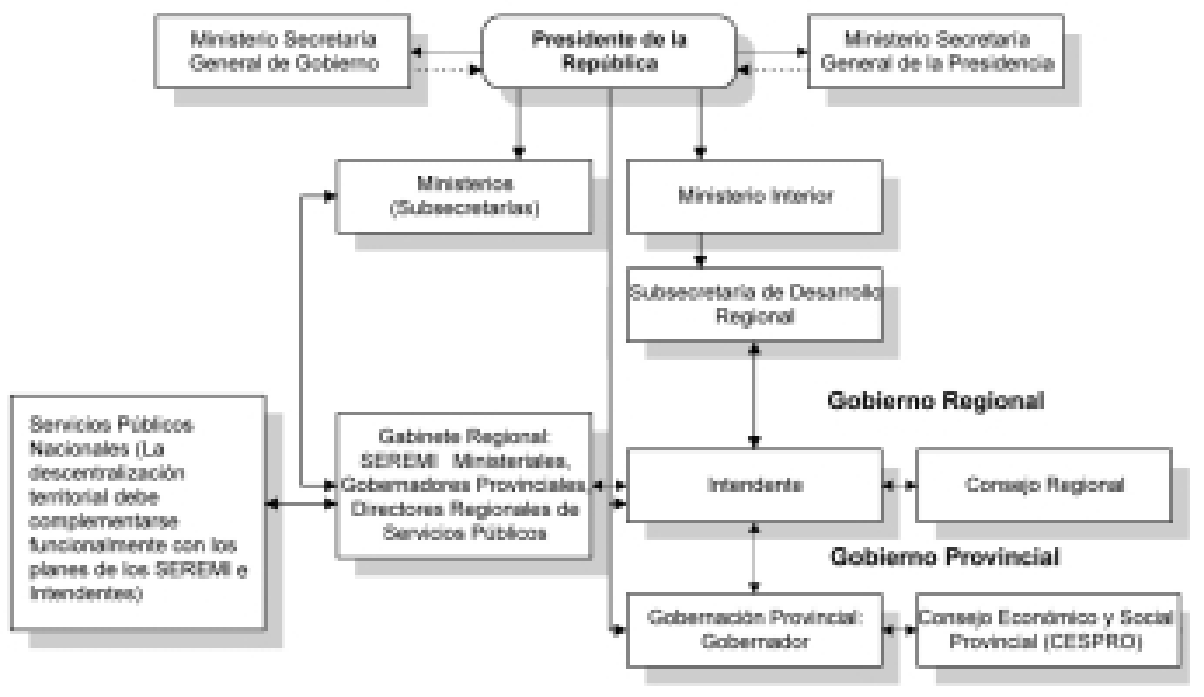

Gobierno y administración de la ciudad de Santiago (Propuesta)

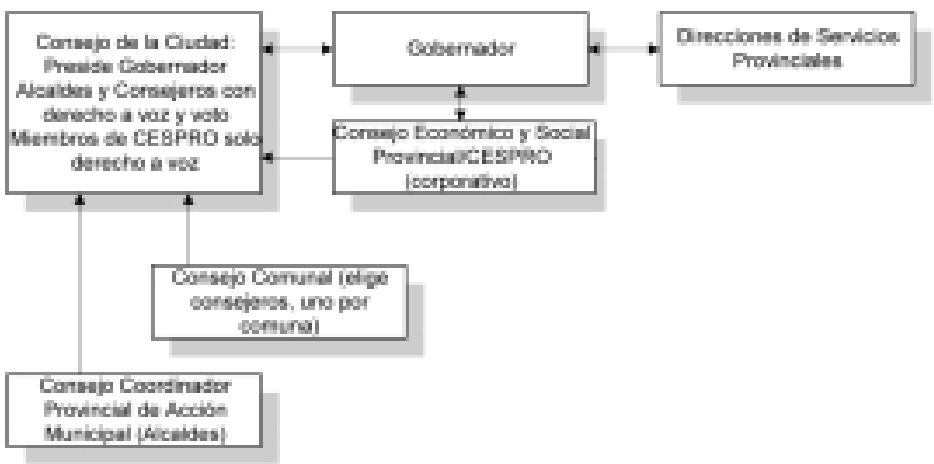


Cabe advertir que, territorialmente, las comunas urbanas de Santiago están comprendidas en la Provincia de Santiago. En consecuencia, desde el punto de vista de ordenamiento territorial del país, la Provincia de Santiago está llamada a ser el fundamento territorial del Gobierno de la Ciudad de Santiago. Reconociéndose ese hecho, probablemente no resultará problemático realizar pequeños ajustes territoriales para efectos administrativos, si se llega a considerar necesario desde el punto de vista técnico (se piensa en casos como el de la Comuna de Puente Alto). En fin, las zonas urbanas son, por naturaleza, entidades dinámicas, y por lo tanto, no susceptibles de ser colocadas en una camisa de fuerza territorial y administrativa: cualquier delimitación territorial de una zona urbana debe ser lo suficientemente amplia y flexible como para adecuarse a la expansión y transformación de las ciudades. De todas formas, siempre será complejo consensuar los términos que designen las zonas metropolitanas. Asumimos la diversidad de definiciones que podemos encontrar sobre éstas, y reconocemos algunos criterios para operacionalizar sanciones estructurantes (vinculación de centros urbanos a través de redes viales), asociativas (utilización en común de la prestación de servicios públicos), etc. Sin embargo, la mejor definición operativa será aquella que no encasille artificialmente la dinámica temporal y espacial que trae consigo el crecimiento, despliegue y desarrollo de una metrópolis.

El modelo contempla dos instancias de gobierno de la ciudad: el Gobernador de la Provincia de Santiago y el Consejo de la Ciudad de Santiago. El Gobernador pasa a ser un poder ejecutivo para el gobierno y administración de la ciudad de Santiago. Es decir, en el caso de la Región Metropolitana, se entenderá que la función del Gobernador es atender la buena administración y gestión del gobierno urbano, que en términos territoriales corresponde al de la Provincia de Santiago. Lo anterior no implica desvincular al Gobernador del Intendente, por cuanto él sigue participando en el gabinete del segundo, y es una autoridad de jerarquía menor a nivel regional. Pero hace necesario dotar al Gobernador de instrumentos jurídicos, económicos y administrativos para que se pueda dedicar a la administración de la ciudad con cierta autonomía.

De este modo, en esencia, no se altera mayormente la institucionalidad vigente, por cuanto el Intendente continúa siendo la máxima autoridad político-administrativa en la región, y el Gobernador una autoridad subordinada, pero con mayor autonomía respecto a sus tareas propias de administración y gobierno urbano. Al Gobernador, en definitiva, compete la buena administración de su provincia urbana dentro de la región.

El Consejo de la Ciudad es el órgano co-responsable, junto con el Gobernador, para la buena administración de la provincia urbana, y con el objeto de poder cumplir esa función estará dotado de alto grado de representación. Al ser un órgano principalmente de representación, el Consejo contribuirá a una mayor coordinación entre inputs sociales urbanos y outputs desde el gobierno metropolitano. La ciudadanía pasará a ser co-responsable de la gestión, mediante la participación de sus representantes en los procesos de decisión, y esta circunstancia mejorará la sintonía entre sus demandas y las respuestas de las entidades del gobierno urbano.

Con la figura del Consejo estamos hablando de la creación de una nueva entidad dentro de la organización política del Estado. Pero ésta se nutre de los elementos que ofrece la institucionalidad vigente.

El Consejo de la Ciudad estaría integrado por el Gobernador, quien lo presidiría, por el Consejo Provincial del Gobernador, por el Consejo Coordinador Regional de Acción Municipal, compuesto 
por los alcaldes, y por concejales elegidos por los miembros del Consejo Municipal de cada comuna de la Provincia de Santiago (uno por consejo).

El Consejo Económico y Social Provincial, actualmente en existencia, podría mantener su carácter de órgano colegiado asesor del Gobernador en el cumplimiento de sus funciones en la Provincia. Sus miembros participarían además en el Consejo de la Ciudad sólo con derecho a voz. Tal circunstancia encuentra fundamento en el hecho que los miembros del Consejo del Gobernador son representantes de sectores corporativos de la sociedad, tales como Fuerzas Armadas, y el Consejo de la Ciudad, en cambio, tiene por función esencial representar a la ciudadanía en la gestión de administración y gobierno urbano. En vistas a sus características corporativas, posiblemente sería preferible que el CESPRO dejara de existir; esto además del hecho de que en la práctica los CESPRO en general no han funcionado efectivamente. Al tener una participación directa en el Consejo de la Ciudad se puede correr el riesgo de captura de los representantes elegidos por parte de grupos con derecho adquirido a tener acceso privilegiado a los agentes de toma de decisión.

El Consejo Coordinador Regional de Acción Municipal, que según la ley 19.175 debiera estar integrado por los alcaldes de la Región Metropolitana, y presidido por el Alcalde de la Comuna de Santiago, puede continuar funcionando dentro del Consejo de la Ciudad, o bien desaparecer, puesto que sus funciones pasan a ser subsumidas en el propio Consejo de la Ciudad. En todo caso, ese Consejo Coordinador pasaría a ser provincial en lugar de regional, es decir, estaría compuesto exclusivamente por los alcaldes de las comunas de la Provincia de Santiago. Si se mantiene como Consejo Coordinador Provincial de Municipalidades, dentro del Consejo de la Ciudad, pasa a estar presidido por el Gobernador de la Provincia de Santiago, de igual modo que si sigue funcionando con independencia. La presidencia del Gobernador se justifica, porque el Gobernador tiene por función central la administración de toda la Provincia Urbana de Santiago.

El Alcalde de la Ciudad de Santiago no preside más el Consejo Coordinador Regional de Acción Municipal, en caso de continuar existiendo como órgano reformado. El Alcalde de Santiago pasa a ser un alcalde comunal, con el objeto que se dedique a su comuna y no a la ciudad. Además, para efectos de coordinación y planificación intercomunal urbana se evita una figuración sobredimensionada del alcalde de la comuna de Santiago.

En cierto sentido, esta parte de la propuesta pone en marcha el actual andamiaje institucional que extraemos de la LOC 19.175, que en su articulado 103 y 104 ordena explícitamente a los servicios públicos, gobiernos regionales y municipalidades a actuar coordinadamente en la formulación de los planes y ejecución de los programas vinculados a la infraestructura social básica y al equipamiento urbano de las áreas metropolitanas, entendiéndose por éstas las extensiones territoriales formadas por dos o más centros de población unidos entre sí por espacios construidos, compartiendo elementos de infraestructura y servicios urbanos. Este es uno de los lugares en que la normativa vigente ha provocado confusión. Se establece que en las áreas metropolitanas, los Gobiernos regionales serán los que ejerzan las atribuciones especificadas sobre los servicios públicos que les confiere la ley. La Región Metropolitana con la Intendencia y sus autoridades como líderes naturales aparecen cristalizadas y jurídicamente avaladas en el artículo 103. Sin embargo, la inacción del Consejo Coordinador Regional de Acción Municipal descrito en el Artículo 104 , entrega un caudal reflexivo interesante. Quizás, lo que explica el que no se haya logrado 
constituir efectivamente radique en que la presidencia deberá materializarse en el edil de la comuna donde se afinque la cabecera regional o provincial, es decir, en el caso de la Región Metropolitana sería la Municipalidad de Santiago. Nuestra propuesta evita los problemas políticos que se podrían derivar de asignarle tanto protagonismo al Alcalde de la comuna de Santiago, al establecer que el Consejo de la Ciudad es presidido por el Gobernador de la Provincia de Santiago. Los alcaldes de las comunas de la Provincia Urbana de Santiago, quienes integrarían el Consejo Coordinador Provincial de Municipalidades, serían miembros plenos del Consejo de la Ciudad con derecho a voz, a integrar comisiones, y a voto, por su calidad de ser representantes populares directamente elegidos en sus comunas.

Finalmente, los concejales elegidos por sus pares, en razón de uno por cada concejo municipal de la Provincia de Santiago, también son miembros plenos del Consejo de la Ciudad con derecho a voz, a integrar comisiones, y a voto, por la misma razón.

Los alcaldes y los representantes de concejales serán los miembros más representativos del Consejo de la Ciudad, y su participación en este consejo promovería un mayor grado de accountability del gobierno urbano, en tanto se verían en la obligación de explicar a los ciudadanos de su comuna por qué se tomaron determinadas decisiones a nivel del gobierno de la ciudad. Su re-elección a nivel municipal estaría, de alguna manera, condicionada por la efectividad del gobierno urbano del que participarían. De este modo, nuestra propuesta de gobierno, administración y gestión de la Ciudad de Santiago, hace una contribución a incrementar el grado de responsabilidad de las políticas generales para el desarrollo de la ciudad. Asimismo, se derivaría un poderoso respaldo político a las iniciativas de esta institucionalidad al estar integradas por representantes elegidos democráticamente.

En nuestro modelo, el Consejo de la Ciudad abordaría distintos temas que le competen en cuanto órgano político-administrativo de la ciudad, mediante la constitución de comités o comisiones de estudio, que elaborarían proposiciones para ser votadas en el pleno del Consejo bajo condición de determinado quórum de los miembros con derecho a voto. El Gobernador, quien sería la máxima autoridad provincial y la primera autoridad administrativa de la ciudad, y quien presidiría el Consejo de la Ciudad, informaría al mismo de las actividades y políticas de su competencia que se llevasen a cabo bajo su jurisdicción, y tendría la atribución especial de dirimir en caso de producirse empates en las votaciones en ese órgano. Asimismo, el Gobernador tendría la responsabilidad de transmitir al Intendente las propuestas y decisiones que se adoptasen respecto a la Provincia Urbana de Santiago, y el Intendente tendría la prerrogativa de aprobar, rechazar o enviar indicaciones al Consejo de la Ciudad.

El Consejo Regional, que representa las comunas de toda la Región Metropolitana, en este modelo no toma parte en el proceso de toma de decisiones respecto al gobierno de la Ciudad de Santiago, y por lo tanto, alteraciones en su composición u origen de sus integrantes no afectarían necesariamente al gobierno urbano. Evidentemente es posible que surjan conflictos de orden político 0 administrativo entre los niveles de gobierno regional y urbano. Por esta razón, las funciones y atribuciones del Gobernador y del Consejo, en cuanto autoridades político-administrativas del Gobierno de Santiago, deben estar claramente delimitadas de las atribuciones y funciones que competen al Intendente y al Consejo Regional, en cuanto autoridades regionales, como así también de las atribuciones y funciones desempeñadas por otras autoridades nacionales, regionales 
y locales. Asimismo es necesario que queden especificados todos los procedimientos de elección y representación del Gobierno de la Ciudad ${ }^{25}$. Por lo tanto será necesario introducir las modificaciones pertinentes en la legislación vigente. En fin, cualquier alteración institucional puede acarrear disputas políticas. El objetivo debe ser que estas disputas sean públicas para que la ciudadanía, debidamente informada, pueda ejercer su derecho democrático para evaluar la gestión de todas las unidades políticas y administrativas correspondientes. En este sentido, aunque no se quiere desconocer que puedan suscitarse disputas políticas al interior del gobierno urbano, 0 entre éste y otros niveles, nuestro modelo permitiría sacar provecho de tales situaciones en tanto accountability y participación ciudadana.

\section{CONCLUSIÓN}

Cabe reiterar que la propuesta de gobierno urbano descrita no tiene la pretensión de ofrecer una solución a todos los problemas de gobernabilidad y de calidad de vida en Santiago. Pero, sin perjuicio del debate sobre aspectos específicos relacionados con la propuesta, tales como modificaciones legales necesarias y toma de conciencia sobre posibles áreas de fricción, costos y presupuesto, en nuestra opinión, con la implementación de esta propuesta, o una similar, se podrían alcanzar cuatro metas relevantes: deslinde de atribuciones y funciones entre autoridades político-administrativas; fortalecimiento del gobierno de la Ciudad de Santiago en relación al gobierno regional; mayor eficacia en la gestión político-administrativa, en términos de coordinación; y mayor representatividad y responsabilidad de los órganos político-administrativos de la ciudad de Santiago.

En todo caso, a pesar de que existe una relativo acuerdo en tanto las deficiencias del gobierno de las grandes ciudades en Chile, son pocas las propuestas de reforma institucional que han emergido. Este artículo busca, al menos, inducir un debate más propositivo respecto de esta temática, el que incluya no sólo imperativos técnicos, sino también consideraciones de viabilidad política, y de profundización democrática.

\section{REFERENCIAS}

Ábalos, José. 1994. La descentralización en Chile: Antecedentes históricos y reformas actuales. Santiago: Instituto de Estudios Urbanos.

Acevedo, José. 1998. Empresa Metropolitana de Residuos (EMERES), en http://www.undp.org/ppp/gln/publications/limanew3-4.htm

Ahumada, Jaime. 1998. "El proceso de descentralización”. En Chile en los noventa, editado por Cristián Toloza y E. Lahera. Santiago: Dolmen, 90-128.

Arriagada, Irma y Lorena Godoy. 2000. "Prevenir o reprimir: Falso dilema de la seguridad ciudadana". Revista de la Cepal 70: 107-132.

Aylwin, Arturo. 1991. "Interrogantes y planteamientos sobre un gobierno metropolitano para Santiago de Chile". Revista Eure 17 (52/53): 143-156.

25 Así, por ejemplo, se deberá especificar en la ley que los concejales podrán elegir en sus comunas a un representante para integrar el Consejo de la Ciudad, y bajo qué condiciones participarín en el mismo. Asimismo se deberá especificar el procedimiento de elección e introducir las modificaciones que sean del caso en la legislación. Por ejemplo, en el caso de producirse un empate en la elección de representantes de concejales en un municipio podría ser el Alcalde quien dirima. 
Blanke, Bernhard y Henning Schridde. 1999. "Bürgerengagement und Aktivierender Staat". Aus Zeitgeschihte und Politik 2425: $3-12$.

Boisier, Sergio. 1994. "Crisis y Alternativas a los procesos de Regionalización”. Revista de la Cepal 52: 179-190.

Boisier, Sergio. 1995. La modernización del Estado. Una mirada desde las regiones, en http://ladb.unm.edu/econ/content/ nuesoc/1995/september/modernizacion.html.

Borja, Jordi y Manuel Castells. 1996. Local y global: la gestión de las ciudades en la era de la información. Estambul: United Nations Center for Human Settlements.

Borja, Jordi. 1995. Barcelona: un modelo de transformación urbana. Quito: Programa de Gestión Urbana.

Cataldo, Jaime. 1991. "Municipalidad de Santiago: Participación ciudadana en la definición de lineamientos para el desarrollo comunal". Revista EURE 17 (52/53): 107-125.

Centro de Estudios y Asistencia Legislativa. 1993. Manual de Gestión Regional. V Región: Ministerio de Planificación y Cooperación.

Fundación Friedrich Ebert. 1994. Manual para la Gestión Regional. Santiago: Fundación Friedrich Ebert.

Centro de Estudios Cordillera. 1992. El Sistema municipal chileno: problemas, desafíos y propuestas. Santiago: Centro de Estudios Cordillera.

Cifuentes, Mercedes y Cristián Larroulet. 1998. El Gobierno de las personas. Santiago: Instituto Libertad y Desarrollo.

Constitución Política de la República de Chile, en http://www.georgetown.edu/pdba/Constitutions/Chile/chile97.html

Daher, Antonio. 1991. Regiones metropolitanas binacionales en el Mercosur, en http://www.scielo.cl/scielo.php?script=sci _arttext\&pid=S0250-71612000007800005\& Ing=en\&nrm=iso\&tlng=es

Dockendorff V., Eduardo. 1992. Metropolización y gestión urbana. Santiago: CED.

Finot, Iván. 1997. Descentralización del Estado y gestión social, análisis y perspectivas. Santiago: ILPES.

Finot, Iván. 1998. Descentralización del Estado y participación ciudadana en América Latina: Un enfoque crítico. Santiago: ILPES. Flores, Verónica y Sofía López. 1997. Santiago ¿qué ciudad queremos? Santiago: Pontificia Universidad Católica de Chile.

Godoy A., Oscar. 1999. "La transición a la democracia en Chile: Pactada". Estudios Públicos 74: 79-106.

Góngora, Mario. 1981. Ensayo histórico sobre la noción de Estado en Chile en los siglos XIX y XX. Santiago: Ediciones la Ciudad. Gross, Patricio. 1989. “Santiago de Chile: 1925-1990. Planificación urbana y modelos políticos”. Revista Eure 17(52/53): 25-37. Gutiérrez B., Hernán y Claudio Rojas M. 1999. "La institucionalidad pública y su contribución al desarrollo regional en el marco de la globalización". Revista de Ciencia Política, Vol. 20 (1): 126-151.

Haeussermann, Hartmut. 2000. "Die Krise der sozialen Stadt". Aus Politik und Zeitgeschichte 10-11: 13-21.

Hernández Olmedo, Luis. 1995. "Complejidades y Desafíos actuales de la descentralización en un Estado unitario". En Chile, Ruta al Tercer Milenio, editado por José A. Ábalos. Santiago: Itesa, 189-224.

Instituto Latinoamericano y del Caribe de Planificación Económica y Social (ILPES). 1994. Modernización y gestión regional: EI caso chileno. Santiago: ILPES.

Jordán, Pablo. 1998. Ciudades Intermedias de América Latina y el Caribe: Propuestas para la gestión urbana. CEPAL/Ministerio degli affari esteri cooperaziones italiano (MAE).

Lefevre, Henri. 1985. De lo rural a lo urbano. Madrid: Península.

Legasso, Victoria. 1993. Gobierno local y políticas sociales en el Gran Santiago. Santiago: MIDEPLAN.

Ley Orgánica Constitucional de Municipalidades, en http://www.colegioabogados.org/normas/leyes/18695-Municipalidades.htm.

Ley Orgánica Constitucional de Bases Generales de la Administración Interior del Estado, en http://www.bcn.cl/pags/legislacion/ leyes/locbga1.htm.

Ley Orgánica Constitucional sobre Gobierno y Administración Regional,en http://www.dipres.cl/control gestion/PMG/ ArticN61_LeyN_19175.pdf.

Ley de Bases Generales del Medio Ambiente, en http://www.ima.ufro.cl/diplomados/diplomado/documentos/Conama/seia/ gestionambiental/ley19300.htm.

Ley General de Urbanismo y Construcción, en http://www.conama.cl/coain/article-15983.html.

Lira, Luis y Fernando Marinovic.1999. Estructuras participativas y descentralización: El caso de los Consejos Regionales en Chile. Santiago: ILPES/CEPAL. 
Martínez, Juan. 1991. El crecimiento poblacional en las grandes urbes. Madrid: Aguilar.

Martner, Gonzalo. 1993. Descentralización y modernización del Estado. Santiago: LOM.

Mény, Yves. 1997. Políticas públicas y teoría del Estado. Santiago: ILPES.

Ministerio de Planificación Nacional (MIDEPLAN). 1996. Balance de seis años de las políticas públicas 1990-1996. Santiago: MIDEPLAN.

Noguera A., Humberto. 1993. El gobierno regional en Chile y la experiencia comparativa. Santiago: Andrés Bello.

PARTICIPA. 1993. El gobierno y la administración interior del Estado: Región, provincia y comuna. Santiago: Participa.

Rodríguez, Alfredo y Fabio Velásquez. 1994. Municipios y servicios públicos: Gobiernos locales en ciudades de América Latina. Santiago: Sur.

Palma, Eduardo. 1995. "Descentralización y democracia: El nuevo municipio latinoamericano". Revista de la CEPAL 52: 41-54. Sabatini, Francisco. 1995. "Paradoja de la planificación urbana en Chile". Revista Eure 21 (62): 61-73.

Sabatini, Francisco. 1997. Liberalización de los mercados de suelo y segregación social en las ciudades latinoamericanas. Santiago: Pontificia Universidad Católica de Chile.

Salazar, Gabriel y Jorge Benítez. 1998. Autonomía, espacio y gestión: el municipio cercenado. Santiago de Chile: Universidad ARCIS. Schmidt, Manfred. 1995. Demokratietheorien. Opladen: UTB.

Valdivieso, Patricio y Juan Enrique Vargas. 2003. “Cambios en el sistema de justicia y sociedad civil en Chile (1990-2002)”. En Instituto de Estudios Comparados en Ciencias Sociales y Penales. Justicia y Sociedad Civil. Buenos Aires: Verlap S.A., 149-244.

Von Haldenwang, Christian. 1990. "Hacia un concepto politológico de la descentralización del Estado en América Latina". Revista Eure 16 (50): 61-77.

Tomás Chuaqui H. es Doctor (Ph. D.) en Ciencia Política, Universidad de Princeton (EE.UU.). Sus áreas de investigación incluyen Teoría Política, Historia del Pensamiento Político y Ética Aplicada. Ha impartido docencia en Chile y Estados Unidos en el área del pensamiento político occidental. Actualmente es Director del Instituto de Ciencia Política de la Pontificia Universidad Católica de Chile.

(E-mail: tchuaqui@puc.cl)

Patricio Valdivieso es Doctor (Ph. D.) de la Katholische Universität Eichstätt (Alemania). Actualmente, es Profesor Adjunto Ordinario del Instituto de Ciencia Política de la Pontificia Universidad Católica de Chile y es investigador responsable del proyecto Fondecyt № 1030584, Educación Política en Justicia (http://www.puc.cl/icp/eticapolitica/).

(E-mail: pvaldivf@puc.cl) 